\title{
Correction: Cortisol reactivity in patients with anorexia nervosa after stress induction
}

Ileana Schmalbach (1D, Benedict Herhaus, Sebastian Pässler, Sarah Runst, Hendrik Berth, Silvia Wolff-Stephan and Katja Petrowski

Correction to: Translational Psychiatry (2020) 10:275 https://doi.org/10.1038/s41398-020-00955-7 published online 10 August 2020

Due to an error in typesetting, the values in Table 2 were not placed in the correct columns. This has now been corrected in the online and PDF versions of the article.
Some minor corrections to references have also been made. The authors apologize for the mistakes. The original article has been corrected.

Published online: 08 April 2021 